\title{
CAVITATION SOLUTIONS \\ TO HOMOGENEOUS VAN DER WAALS TYPE FLUIDS \\ INVOLVING PHASE TRANSITIONS
}

BY

\section{BAISHENG YAN}

School of Mathematics, University of Minnesota, Minneapolis, Minnesota

Abstract. In this paper, weak solutions to some special Cauchy problems involving phase transitions in $\mathbf{R}^{3}$ are constructed. These solutions exhibit the point singularity known as cavitation.

1. Introduction. In this paper, we prove the nonuniqueness of the following special Cauchy problem for certain parameters $\lambda>0$ in a ball $B_{\rho} \subset \mathbf{R}^{3}$ :

$$
\mathbf{u}_{t t}=\nabla \cdot \mathscr{S}(\nabla \mathbf{u})
$$

in $B_{\rho} \times[0, T]$, with the homogeneous boundary condition:

$$
\mathbf{u}(\mathbf{x}, t)=\lambda \mathbf{x}, \quad \mathbf{x} \in \partial B_{\rho}, \quad t \in[0, T] ;
$$

and the initial condition:

$$
\mathbf{u}(\mathbf{x}, 0)=\lambda \mathbf{x}, \quad \mathbf{x} \in B_{\rho}, \quad \mathbf{u}_{t}(\mathbf{x}, 0)=0, \quad \mathbf{x} \in B_{\rho} .
$$

The stress tensor $\mathscr{S}(F)$ is defined by $\varphi(\operatorname{det} F)(\operatorname{adj} F)^{\mathrm{T}}$ and $\varphi: \mathbf{R}^{+} \rightarrow \mathbf{R}$ has a graph as shown in the van der Waals fluid that decreases in some transition interval. (See Fig. 1.) This type state function can also be a model in phase transitions in onedimensional elastodynamics. For instance, the intervals where $\varphi$ increases represent two different phases that are separated by the transition interval; see, e.g., [4], [7], and [10].

Note that Eq. (1.1) can be written in terms of conservation laws of the material in Lagrangian reference:

$$
\left\{\begin{array}{l}
\mathbf{v}_{t}(\mathbf{x}, t)=\nabla \cdot \mathscr{S}(F(\mathbf{x}, t)), \\
F_{t}(\mathbf{x}, t)=\nabla \mathbf{v}(\mathbf{x}, t) \text { for } \mathbf{x} \in B_{\rho} \text { and } t>0,
\end{array}\right.
$$

where $\mathbf{v}(\mathbf{x}, t)=\mathbf{u}_{t}(\mathbf{x}, t), F(\mathbf{x}, t)=\nabla \mathbf{u}(\mathbf{x}, t)$ and $\mathbf{u}(\mathbf{x}, t)$ represent the velocity, deformation gradient, and deformation at time $t$ of the fluid occupying the point $\mathbf{x} \in B_{\rho}$ in the reference configuration, respectively. 
The uniqueness of the problem (1.1)-(1.3) under certain ellipticity conditions on $\mathscr{S}(F)$ has been well known (see, e.g., Wheeler [12]). The stress tensor $\mathscr{S}(F)$ we consider in this paper violates these ellipticity conditions very badly. It is natural that the uniqueness should fail for (1.1)-(1.3). Obviously, the homogeneous equilibrium defined by $\mathbf{u}_{\lambda}(\mathbf{x}) \equiv \lambda \mathbf{x}$ is a classical solution to (1.1)-(1.3) for any $\lambda>0$.

The aim of this paper is to construct weak solutions to (1.1)-(1.3) for certain parameters $\lambda>0$ that exhibit certain prescribed point singularities.

We use the same method as that used in [9] to construct weak solutions with point defects. This type of defect is known as cavitation. Let us mention that in nonlinear elasticity theory, cavitation can happen for certain materials such as liquid crystals (see [6]).

Like solutions constructed in [9], solutions we construct in $\S 4$ are also of the form

$$
\mathbf{u}(\mathbf{x}, t)=t f(|\mathbf{x}| / t) \mathbf{x} /|\mathbf{x}|, \quad \mathbf{x} \neq 0, \quad t>0,
$$

where $f(s)$ is a continuous, piecewise $C^{2}$ function, and satisfies $f(0)>0$ and $f(s)=\lambda s$ for $s \geq \sigma$, for some $\sigma>0$. We also assume that $\mathbf{u}(\mathbf{x}, t)=\lambda \mathbf{x}$ when $t=0$ and $\mathbf{x} \in B_{\rho}$, and $\mathbf{u}(\mathbf{x}, t)=0$ when $\mathbf{x}=0$ for all $t>0$. At each time $t \in(0, \rho / \sigma)$ such solutions differ from the trivial solution $\mathbf{u}_{\lambda}$ only in the ball $B_{t \sigma}$. We shall thus call this ball the transition region. We remark that inside the transition region, the solutions open a hole at $\mathbf{x}=0$; thus such solutions will be called cavitation solutions to (1.1)-(1.3).

We remark that since the assumptions on $\mathscr{S}(F)$ and $\varphi(v)$ are different from those of [9], results of this paper differ in many physically related aspects. We shall see that these solutions undergo phase transitions and some of the solutions contain shocks inside the transition region (see $\S 4$ for details). Furthermore, in general, the energy reduction criterion (see, e.g., Dafermos [2], DiPerna [3], James [7], and Lax [8]) fails for most of the solutions when phase transition occurs. On the other hand, the energy reduction criterion can still hold even when a full phase transition does occur, mainly due to interactions and cancellations among the shocks; see $\S 5$ for details.

2. Constitutive assumptions. Suppose $\varphi: \mathbf{R}^{+} \rightarrow \mathbf{R}$ is a $C^{2}$ function and there exist $0<A<\alpha<B<\beta<C$ such that (cf. Fig. 1)

(A) $\varphi(v)=0$ for $v=A, B, C$ and $\varphi^{\prime}(\alpha)=\varphi^{\prime}(\beta)=0$;

(B) $\varphi(v)$ is increasing for $0<v<\alpha$ and $v>\beta$;

(C) $\varphi^{\prime \prime}(v)<0$ for $v \in(A, B) \cup(C,+\infty)$; and

(D) $\lim _{v \rightarrow 0^{+}} \varphi(v)=-\infty$ and $\lim _{v \rightarrow+\infty} \varphi(v)=+\infty$.

Let us define $\Psi(v, w)=(\varphi(v)-\varphi(w)) /(v-w)$ for $v \neq w$ and $\Psi(v, w)=\varphi^{\prime}(v)$ for $v=w$.

By assumptions (A)-(D), one can see that there exists a unique $v^{*} \in(C,+\infty)$ such that $\varphi^{\prime}\left(v^{*}\right)=\Psi\left(v^{*}, \alpha\right) \equiv \nu>0$. Define now

$$
L(v)=\min \left\{\Psi(v, \alpha) ; \Psi\left(v, v^{*}\right)\right\} .
$$

Then for any $w \in(\alpha, B]$ and $0 \leq q<\nu$, and $v \in[A, \alpha)$ and $0 \leq p<L(v)$ one can define functions $r(w, q) \in[C,+\infty)$ and $m_{1}(v, p) \in(\alpha, B]$ and $m_{2}(v, p) \in$ 


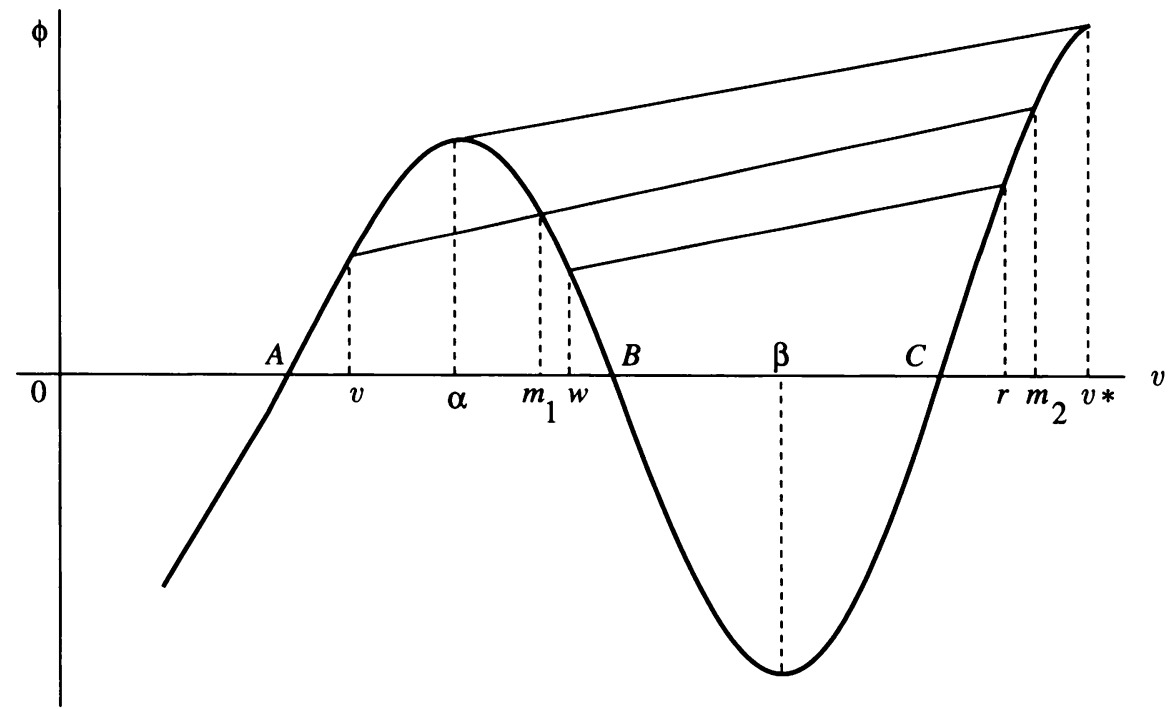

FIG. 1. The graph of $\phi=\varphi(v)$. Here $r=r(w, q)$ and $m_{i}=$ $m_{i}(v, p)$.

$\left[C, v^{*}\right)$ such that

$$
\Psi(r(w, q), w)=q, \quad \Psi\left(m_{i}(v, p), v\right)=p .
$$

Note that $0<q<\varphi^{\prime}(r(w, q))$ as a consequence of (2.2).

REMARK 2.1. As in some phase transition theories, see [4], [7], and [10], a deformation gradient or a phase $F$ is said to be in the $\alpha$-phase if $\operatorname{det} F \in(0, \alpha)$, in the $\beta$-phase if $\operatorname{det} F \in(\beta,+\infty)$, and a phase mixture if $\operatorname{det} F \in(\alpha, \beta)$.

3. Admissible solutions. In this section we follow [9]. We also refer to this paper and Ball [1] for proof of many results of this section.

Let $\Omega \subset \mathbf{R}^{3}$ and define

$$
\mathscr{A}_{p}(\Omega) \equiv\left\{\mathbf{y} \in W^{1, p}\left(\Omega ; \mathbf{R}^{3}\right) \mid \operatorname{det} \nabla \mathbf{y}(\mathbf{x})>0 \text { a.e. } \mathbf{x} \in \Omega\right\} .
$$

Given $T>0$, we call $\mathbf{u}:[0, T] \rightarrow \mathscr{A}_{p}(\Omega)$ an admissible motion of duration $T$ if $\mathbf{u} \in C\left([0, T] ; W^{1, p}\left(\Omega ; \mathbf{R}^{3}\right)\right) \cap C^{1}\left([0, T] ; L^{p}\left(\Omega ; \mathbf{R}^{3}\right)\right)$. A motion $\mathbf{u}(\mathbf{x}, t)$ is called a weak solution to problem (1.1)-(1.3) if $\mathbf{u}$ satisfies (1.3) for almost every $\mathbf{x} \in \Omega$ and u satisfies $(1.2)$ in the sense of trace and $\mathscr{S}(\nabla \mathbf{u}(\cdot, t)) \in L_{\mathrm{loc}}^{1}(\Omega)$ for every $t \in[0, T]$ and, moreover, for all $\psi \in C_{c}^{\infty}\left(\Omega_{T}\right)$ with $\Omega_{T}=\Omega \times(0, T)$,

$$
\iint_{\Omega_{T}}\left(\mathbf{u}_{t} \psi_{t}-\mathscr{S}(\nabla \mathbf{u}) \nabla \psi\right) d \mathbf{x} d t=0
$$

It is easy to see that the equilibrium deformation $\overline{\mathbf{u}}_{\lambda}(\mathbf{x}, t) \equiv \lambda \mathbf{x}$ for $(\mathbf{x}, t) \in \bar{\Omega}_{T}$ is always a weak solution of (1.1) in the sense of this definition for any $\lambda>0$. The uniqueness of this solution under some conditions has been proved by [12]. In this paper, we shall prove that (1.1) possesses weak solutions other than $\overrightarrow{\mathbf{u}}_{\lambda}(\mathbf{x}, t)$ for some $\lambda>0$. These solutions exhibit some point defects in the material as we shall see in the sequel, and thus be termed as cavitation solutions. Existence of such 
solutions, of course, depends on our admissible class of motions since here we only allow $1 \leq p<3$ in $(3.1)$.

Now let $\Omega \equiv B_{\rho}=\left\{\mathbf{x} \in \mathbf{R}^{3}|| \mathbf{x} \mid<\rho\right\}$ and consider a radial function $\mathbf{y}: B_{\rho} \rightarrow \mathbf{R}^{3}$ defined by $\mathbf{y}(\mathbf{x})=r(|\mathbf{x}|)(\mathbf{x} /|\mathbf{x}|)$ for $\mathbf{x} \neq 0$, where $r:[0, \rho) \rightarrow[0, \infty)$. The following results have been proven in [1] and [9].

Lemma 3.1. Let $\mathbf{y}(\mathbf{X})$ be defined as above. Then $\mathbf{y} \in \mathscr{A}_{p}\left(B_{\rho}\right)$ if and only if $r$ is absolutely continuous on $(0, \rho), r^{\prime}(R)>0$ for a.e. $R \in(0, \rho)$ and

$$
\int_{0}^{\rho}\left[\left|r^{\prime}(R)\right|^{p}+\left|\frac{r(R)}{R}\right|^{p}\right] R^{2} d R<+\infty .
$$

In this case the deformation gradient of $\mathbf{y}$ is given by

$$
\nabla \mathbf{y}(\mathbf{x})=(r(R) / R) I+\left[r^{\prime}(R)-r(R) / R\right](\mathbf{x} / R) \otimes(\mathbf{x} / R)
$$

for a.e. $\mathbf{x} \in B_{\rho}$, where $R=|\mathbf{x}|$.

In what follows, we call a function $\mathbf{u}: \Omega_{T} \rightarrow \mathbf{R}^{3}$ a cavitation function if

$$
\mathbf{u}(\mathbf{x}, t)= \begin{cases}0 & \text { for } \mathbf{x}=0 \text { and } t>0, \\ \lambda \mathbf{x} & \text { for } \mathbf{x} \in B_{\rho} \text { and } t=0, \\ t f(|\mathbf{x}| / t) \mathbf{x} /|\mathbf{x}| & \text { for } \mathbf{x} \neq 0 \text { and } t>0\end{cases}
$$

for some $f:[0, \infty) \rightarrow[0, \infty)$ satisfying $f(s)=\lambda s$ for $s \geq \sigma \equiv \rho / T$.

Lemma 3.2. Let $\mathbf{u}$ be defined by (3.3) and suppose $1 \leq p<3$. If $f$ is a continuous piecewise $C^{1}$ function satisfying $f^{\prime}(s)>0$ for a.e. $s>0$, then $\mathbf{u}$ is an admissible motion and in this case

$$
\begin{gathered}
\mathbf{u}_{t}(\mathbf{x}, t)=\left(f(s)-s f^{\prime}(s)\right) \mathbf{x} /|\mathbf{x}|, \quad t>0, \\
\nabla \mathbf{u}(\mathbf{x}, t)=\frac{f(s)}{s} I+\left[f^{\prime}(s)-\frac{f(s)}{s}\right] \frac{\mathbf{x}}{|\mathbf{x}|} \otimes \frac{\mathbf{x}}{|\mathbf{x}|},
\end{gathered}
$$

for a.e. $\mathbf{x} \in B_{\rho}$ and $t \in(0, T]$, where $s=|\mathbf{x}| / t$.

Suppose $\mathbf{u}(\mathbf{x}, t)$ is defined by (3.3). If $f(s)$ is of class $C^{2}$ on $[a, b] \subset[0, \infty)$, then by Lemma 3.2 one can calculate that (see [2]) on the domain $D_{a b} \equiv\{(\mathbf{x}, t) \in$ $\left.\Omega_{T} \mid a<s<b\right\}$, where $s=|\mathbf{x}| / t$,

$$
\begin{gathered}
\mathbf{u}_{t}(\mathbf{x}, t)=(f(s)-s \dot{f}(s)) \mathbf{x} /|\mathbf{x}|, \\
\mathscr{S}(\nabla \mathbf{u}(\mathbf{x}, t))=\varphi(\omega(s))\left[\frac{\dot{f}(s) f(s)}{s} I+\left(\left(\frac{f(s)}{s}\right)^{2}-\frac{\dot{f}(s) f(s)}{s}\right) \frac{\mathbf{x}}{|\mathbf{x}|} \otimes \frac{\mathbf{x}}{|\mathbf{x}|}\right]
\end{gathered}
$$

where $\omega(s) \equiv \dot{f}(s)(f(s) / s)^{2}$ denotes the specific volume of the fluid, and the dot indicates $d / d s$.

From this, the equation in (1.1) on $D_{a b}$ is equivalent to

$$
\left[s^{2}-\varphi^{\prime}(\omega(s))\left(\frac{f(s)}{s}\right)^{4}\right] \ddot{f}(s)=\frac{2}{s} \dot{f}(s) \varphi^{\prime}(\omega(s))\left(\frac{f(s)}{s}\right)^{3}\left(\dot{f}(s)-\frac{f(s)}{s}\right) \text {. }
$$

The following result is similar to the one in [9]. 
THEOREM 3.3. Let $f$ be a continuous function that is piecewise $C^{2}$ on $[0, \infty)$ with all discontinuities in $\dot{f}$ at $0=s_{0}<s_{1}<\cdots<s_{L}:=\sigma$ and let $f$ be of class $C^{2}$ on $\left[s_{i}, s_{i+1}\right]$ for all $i=0,1, \ldots, L-1$. Suppose that $f$ satisfies the following conditions:

(i) $f(s)=\lambda s$ for all $s \geq \sigma$;

(ii) $\dot{f}(s)>0$ for every $s \neq s_{i}, i=0,1, \ldots, L$;

(iii) $\left[s^{2}-\varphi^{\prime}(\omega(s))(f(s) / s)^{4}\right] \ddot{f}(s)=\frac{2}{s} \dot{f}(s) \varphi^{\prime}(\omega(s))(f(s) / s)^{3}(\dot{f}(s)-f(s) / s)$ for every $s \neq s_{i}, \quad i=0,1, \ldots, L$;

(iv) $s_{i}^{4}\left[\dot{f}\left(s_{i}^{+}\right)-\dot{f}\left(s_{i}^{-}\right)\right]=f^{2}\left(s_{i}\right)\left[\varphi\left(\omega\left(s_{i}^{+}\right)\right)-\varphi\left(\omega\left(s_{i}^{-}\right)\right)\right]$for $i=1,2, \ldots, L$; and

(v) $\lim _{s \rightarrow 0^{+}} \varphi(\omega(s))=0$.

Then the function $\mathbf{u}(\mathbf{x}, t)$ defined by (3.3) is a weak solution of $(1.1)$ in $B_{\rho} \times[0, T]$ with $T \equiv \rho / \sigma$.

Proof. The proof is similar to that of Theorem 5.1 of [9], which is based on the divergence theorem. We only make some remarks on the conditions; for details we refer to [9]. By Lemma 3.2, conditions (i) and (ii) assure that $\mathbf{u}(\mathbf{x}, t)$ is an admissible motion; (iv) is the so-called Rankine-Hugoniot jump condition across the shock surface $\partial B_{s_{s} t}$; while $(\mathrm{v})$ makes $\mathscr{S}(\nabla \mathbf{u}(\cdot, t)) \in L^{1}\left(B_{\rho}\right)$ for all $t \in[0, T]$ and this condition also has other meaning for the calculation of the total energy of the solution $\mathbf{u}(\mathbf{x}, t)$ (

In order to solve the equation in (iii) of the theorem, we introduce the following functions:

$$
P(f, \omega ; s)=\frac{\omega s^{2}}{f^{2}}, \quad Q(f, \omega ; s)=\frac{2 s^{5} \omega\left(s^{3} \omega-f^{3}\right)}{f^{3}\left[s^{6}-\varphi^{\prime}(\omega) f^{4}\right]} .
$$

Then one can see that the equation in (iii) is equivalent to the following O.D.E. system:

$$
\dot{f}=P(f, \omega ; s), \quad \dot{\omega}=Q(f, \omega ; s) .
$$

The following result will be frequently used in the next section.

Proposition 3.4. Let $a \geq 0, F>0$ and $W>0$ satisfy $a^{6}-\varphi^{\prime}(W) F^{4} \neq 0$. Then there exist an interval $[a, a+\delta)$ and a unique function $f \in C^{2}\left([a, a+\delta) ; \mathbf{R}^{+}\right)$such that if $\omega(s)=\dot{f}(s)(f(s) / s)^{2}$,

$$
\dot{f}=P(f, \omega ; s), \quad a<s<a+\delta, \quad \dot{\omega}=Q(f, \omega ; s), \quad f(a)=F, \quad \omega\left(a^{+}\right)=W .
$$

Furthermore, if $a^{3} W-F^{3} \neq 0$ then $s^{3} \omega(s)-f(s)^{3} \neq 0$ for any $s \in(a, a+\delta)$.

Proof. The existence is standard as in the general theory of O.D.E. We prove the second part. Suppose that $s_{0}^{3} \omega\left(s_{0}\right)-f\left(s_{0}\right)^{3}=0$ for some $s_{0} \in(a, a+\delta)$. Then $\dot{f}\left(s_{0}\right)=f\left(s_{0}\right) / s_{0}$. Consider the functions $f_{*}(s):=\dot{f}\left(s_{0}\right) s$ and $\omega_{*}(s):=f\left(s_{0}\right)^{3} / s_{0}^{3}$; one can see that they solve (3.6) and $f_{*}\left(s_{0}\right)=f\left(s_{0}\right)$ and $\omega_{*}\left(s_{0}\right)=\omega\left(s_{0}\right)$. Thus a standard uniqueness theorem in O.D.E. (see, e.g., Hale [5]) shows that $f(s)=f_{*}(s)$ and $\omega_{*}(s)=\omega(s)$ for all $s \in\left[a, s_{0}\right)$. This is a contradiction since $a^{3} W-F^{3} \neq 0$. 
4. Construction of cavitation solutions. Let $\mu>0$ be given and suppose one solves the following ordinary differential equation:

$$
\dot{f}=P(f, \omega ; s), \quad \dot{\omega}=Q(f, \omega ; s), \quad f(0)=\mu, \quad \omega\left(0^{+}\right)=A .
$$

By Proposition 3.4, the problem (4.1) can be solved on $[0, \delta)$ for some $\delta>0$. Define $p(s)=s^{6} /\left(f(s)^{4}\right)$ and $l(s)=L(\omega(s))$. Since $p\left(0^{+}\right)=0$ and $l\left(0^{+}\right)>0$, one can find $0<\tau<\delta$ such that

$$
0<p(s)<\frac{2^{6} s^{6}}{f(s)^{4}}<\min \{\nu, l(s)\}, \quad \forall s \in(0, \tau] .
$$

Now one can define the following functions on $(0, \tau]$ :

$$
\begin{gathered}
m_{i}(s)=m_{i}(\omega(s), p(s)), \quad i=1,2 \\
\theta_{1}(s)=p(s)\left[\left(\frac{f(s)}{s}\right)^{3}-m_{2}(s)\right]-\left[\varphi\left(\left(\frac{f(s)}{s}\right)^{3}\right)-\varphi\left(m_{2}(s)\right)\right] .
\end{gathered}
$$

By assumption (D) it follows that $\lim _{s \rightarrow 0^{+}} \theta_{1}(s)=-\infty$. Therefore one can choose $s_{1} \in(0, \tau)$ so that $\theta_{1}(s)<0$ for all $s \in\left[0, s_{1}\right]$.

Case I. There exists $\sigma \in\left(0, s_{1}\right]$ such that $m_{i}(\sigma)=(f(\sigma) / \sigma)^{3}$ for some $i=1,2$.

In this case if one defines $\hat{f}(s)=f(s)$ for $0<s<\sigma$ and $\hat{f}(s)=\frac{f(\sigma)}{\sigma} s$ for $s \geq \sigma$, then by Theorem 3.3 it follows that the function $\hat{f}$ gives a solution to (1.1)-(1.3) through (3.3).

Case II. For any $s \in\left(0, s_{1}\right]$ and $i=1,2$, one has $m_{i}(s) \neq(f(s) / s)^{3}$.

It is easy to see that in this case

$$
m_{1}(s)<m_{2}(s)<(f(s) / s)^{3}
$$

for all $s \in\left(0, s_{1}\right]$. Now we consider the following problem:

$$
\begin{aligned}
\dot{g}(s) & =P(g, w ; s), \quad \dot{w}(s)=Q(g, w ; s), \\
g\left(s_{1}\right) & =f\left(s_{1}\right), \quad w\left(s_{1}\right)=m_{1}\left(s_{1}\right) .
\end{aligned}
$$

Since $s_{1}^{6}-\varphi^{\prime}\left(m_{1}\left(s_{1}\right)\right) f\left(s_{1}\right)^{4}>0$ and $s_{1}^{3} m_{1}\left(s_{1}\right)-f\left(s_{1}\right)^{3}<0$, thus by Proposition 3.4, Eq. (4.4) can be solved on $s \in\left[s_{1}, s_{1}+\delta\right.$ ) for some $\delta>0$. Let $q(s)=s^{6} / g(s)^{4}$. If $\tau \in\left(s_{1}, s_{1}+\delta\right)$ and $\tau<2 s_{1}$ then $0<q(s) \leq\left(2^{6} s_{1}^{6}\right) /\left(f\left(s_{1}\right)^{4}\right)<\nu$ for all $s \in\left[s_{1}, \tau\right]$. Therefore, the following functions will be well-defined on $\left[s_{1}, \tau\right]$ :

$$
\begin{gathered}
r(s)=r(w(s), q(s)), \quad C \leq r(s)<v^{*}, \\
\theta_{2}(s)=q(s)\left[\left(\frac{g(s)}{s}\right)^{3}-r(s)\right]-\left[\varphi\left(\left(\frac{g(s)}{s}\right)^{3}\right)-\varphi(r(s))\right] .
\end{gathered}
$$

Note that since $r\left(s_{1}\right)=m_{2}\left(s_{1}\right)$ and $\theta_{1}\left(s_{1}\right)<0$, we can choose $s_{2} \in\left(s_{1}, \tau\right]$ such that $\theta_{2}(s)<0$ for all $s \in\left[s_{1}, s_{2}\right]$.

Subcase II.1. There exists $\sigma \in\left(s_{1}, s_{2}\right]$ such that $r(\sigma)=(g(\sigma) / \sigma)^{3}$.

In this case, we define

$$
\hat{f}(s)= \begin{cases}f(s) & \text { for } s \in\left[0, s_{1}\right), \\ g(s) & \text { for } s \in\left[s_{1}, \sigma\right), \\ \frac{g(\sigma)}{\sigma} s & \text { for } s \geq \sigma\end{cases}
$$


Then again by Theorem 3.3, this function $\hat{f}$ gives a solution to (1.1)-(1.3).

Subcase II.2. For any $s \in\left(s_{1}, s_{2}\right], r(s) \neq(g(s) / s)^{3}$.

In this case, by (4.3), it follows that $r(s)<(g(s) / s)^{3}$. We now consider the following problem:

$$
\begin{aligned}
\dot{h}(s) & =P(h, v ; s), \quad \dot{v}(s)=Q(h, v ; s), \\
h\left(s_{2}\right) & =g\left(s_{2}\right), \quad v\left(s_{2}\right)=r\left(s_{2}\right) .
\end{aligned}
$$

First of all, it follows from Proposition 3.4 that (4.6) can be solved on $\left[s_{2}, s_{2}+\delta\right.$ ) for some $\delta>0$; using similar arguments as in [9], we can extend the solution to a maximal interval $\left[s_{2}, s_{M}\right)$; and using the same argument and the second part of Proposition 3.4 we can also prove that $s_{M}<+\infty$ and

$$
s_{M}^{6}-\varphi^{\prime}\left(v\left(s_{M}\right)\right) h\left(s_{M}\right)^{4}=0, \quad s^{3} v(s)-h(s)^{3}<0
$$

for all $s \in\left[s_{2}, s_{M}\right)$.

Proposition 4.1. Let $(h(s), v(s)), s \in\left[s_{2}, s_{M}\right)$, be the extended solution to (4.6).

(i) If $s_{M}^{3} v\left(s_{M}\right)-h\left(s_{M}\right)^{3}<0$, then there exists $s_{3} \in\left(s_{2}, s_{M}\right)$ such that

$$
\Psi\left(v\left(s_{3}\right),\left(h\left(s_{3}\right) / s_{3}\right)^{3}\right)=s_{3}^{6} /\left(h\left(s_{3}\right)^{4}\right) .
$$

(ii) If $s_{M}^{3} v\left(s_{M}\right)-h\left(s_{M}\right)^{3}=0$, then $\ddot{h}\left(s_{M}^{-}\right)=0$; thus $h \in C^{2}\left(\left[s_{2}, s_{M}\right]\right)$.

Proof. We first prove (i). This suppose $s_{M}^{3} v\left(s_{M}\right)-h\left(s_{M}\right)^{3}<0$. We consider

$$
\theta_{3}(s)=\frac{s^{6}}{h(s)^{4}}\left[\left(\frac{h(s)}{s}\right)^{3}-v(s)\right]-\left[\varphi\left(\left(\frac{h(s)}{s}\right)^{3}\right)-\varphi(v(s))\right] .
$$

Note that $\theta_{3}\left(s_{2}\right)=\theta_{2}\left(s_{2}\right)<0$. On the other hand, by (4.7) and the mean value theorem it follows that

$$
\theta_{3}\left(s_{M}\right)=\left(\left(\frac{h\left(s_{M}\right)}{s_{M}}\right)^{3}-v\left(s_{M}\right)\right)\left(v\left(s_{M}\right)-\xi_{1}\right) \varphi^{\prime \prime}\left(\xi_{2}\right),
$$

where $C<v\left(s_{M}\right)<\xi_{1}<\xi_{2}<h\left(s_{M}\right)^{3} / s_{M}^{3}$. By the assumption (C) in $\S 2$ one has $\theta_{3}\left(s_{M}\right)>0$; thus by the intermediate theorem, there exists an $s_{3} \in\left(s_{2}, s_{M}\right)$ such that $\theta_{3}\left(s_{3}\right)=0$. This is just the conclusion of part (i). Now we prove case (ii). It suffices to prove that $\lim _{s \rightarrow s_{M}^{-}} \dot{v}(s)=0$. Let $s_{n} \nearrow s_{M}$ be such that

$$
\lim _{n \rightarrow \infty} \dot{v}\left(s_{n}\right)=V:=\limsup _{s \rightarrow s_{M}^{-}} \dot{v}(s) .
$$

By (3.5) and the mean value theorem, for each $n=4,5, \ldots$ we have a $\tau_{n} \in\left(s_{n}, s_{M}\right)$ such that

$$
\dot{v}\left(s_{n}\right)=\frac{2 s_{n}^{5} v\left(s_{n}\right)}{h^{3}\left(s_{n}\right)} \cdot \frac{\tau_{n}^{3}}{\left[6 \tau_{n}^{5}-4 h\left(\tau_{n}\right) \tau_{n}^{2} v\left(\tau_{n}\right) \varphi^{\prime}\left(v\left(\tau_{n}\right)\right)\right]\left(\dot{v}\left(\tau_{n}\right)\right)^{-1}-\varphi^{\prime \prime}\left(v\left(\tau_{n}\right)\right) h^{4}\left(\tau_{n}\right)} .
$$

We claim that the sequence $\left\{\dot{v}\left(\tau_{n}\right)\right\}_{n=4}^{\infty}$ cannot have any subsequence converging to a number $l \in(0,+\infty]$. Suppose it had such a subsequence converging to some 
$l \in(0,+\infty]$. If $l=+\infty$, then $V=+\infty$. On the other hand, in (4.11), letting $n \rightarrow \infty$ we would have $V<+\infty$ since $\varphi^{\prime \prime}\left(v\left(s_{M}\right)\right)<0$. This is a contradiction. If $0<l<+\infty$, again by (4.10) and (4.11), we would obtain

$$
V=\frac{2 s_{M}^{5} l}{2 s_{M}^{5}-\varphi^{\prime \prime}\left(v\left(s_{M}\right)\right) h^{4}\left(s_{M}\right) l}<l
$$

which also contradicts the definition of $V$ being the lim sup; thus the claim holds. Therefore $\lim _{n \rightarrow \infty} \dot{v}\left(\tau_{n}\right)=0$, which combined with (4.11), shows that $V=0$.

Define $\sigma$ to be $s_{3}$ if case (i) of Proposition 4.1 holds, or let $\sigma=s_{M}$ if case (ii) holds. Then define

$$
\hat{f}(s)= \begin{cases}f(s) & \text { for } 0 \leq s<s_{1} \\ g(s) & \text { for } s_{1} \leq s<s_{2} \\ h(s) & \text { for } s_{2} \leq s<\sigma \\ (h(\sigma) / \sigma) s & \text { for } s \geq \sigma .\end{cases}
$$

Then as before it follows from Theorem 3.3 that this $\hat{f}$ gives a solution to (1.1)-(1.3) through (3.3).

Finally, the construction in Case I, or (4.5) in Subcase II.1, or (4.12) in Subcase II. 2 shows the existence of cavitation solutions to (1.1)-(1.3) for any given initial value $\mu>0$ (cf. (4.1)).

5. Exchanges of total energy. For any admissible motion $\mathbf{u}:[0, T] \rightarrow \mathscr{A}_{p}(\Omega)$, define the total energy at time $t \in[0, T]$ by

$$
E(t, \mathbf{u} ; \Omega)=\int_{\Omega}\left(\frac{1}{2}\left|\mathbf{u}_{t}(\mathbf{x}, t)\right|^{2}+\Phi(\operatorname{det} \nabla \mathbf{u}(\mathbf{x}, t))\right) d \mathbf{x} .
$$

Here $\Phi(v)$ is a positive function satisfying $\Phi^{\prime}(v)=\varphi(v)$. In this section we shall discuss exchanges of total energy for cavitation solutions $\mathbf{u}(\mathbf{x}, t)$ constructed in $\S 4$.

The following result is useful in computing the total energy of cavitation solutions; a similar version of the result can be found in [9].

Lemma 5.1. Let $f \in C^{2}\left((a, b) ; \mathbf{R}^{+}\right)$satisfy Eq. (3.4) with $\omega(s)=\dot{f}(s)(f(s) / s)^{2}$ and $0<a<b$. Then the following identity holds in $(a, b)$ :

$$
\begin{aligned}
& s^{2}\left(\frac{1}{2}(f(s)-s \dot{f}(s))^{2}+\Phi(\omega(s))\right) \\
& =\frac{d}{d s}\left[\frac{s^{3}}{3}\left(\frac{1}{2}(f(s)-s \dot{f}(s))^{2}+\Phi(\omega(s))\right)+\frac{f(s)^{3}-s^{3} \omega(s)}{3} \varphi(\omega(s))\right] .
\end{aligned}
$$

Proof. The result follows from a long but direct calculation using (3.4) and (3.6).

Suppose now that $f(s)$ satisfies all the conditions of Theorem 3.3 and let $\mathbf{u}$ be defined by (3.3) and $\omega(s)$ as before. By the lemma above it follows that

$$
E\left(t, \mathbf{u} ; B_{\rho}\right)=\left(4 \pi t^{3} / 3\right)\left[\Phi\left(\lambda^{3}\right)\left(\rho^{3} / t^{3}-\sigma^{3}\right)+\left.\sum_{i=0}^{L-1}\left(s^{3} K(s)\right)\right|_{s_{i}} ^{s_{i+1}}\right],
$$


where

$$
K(s)=\Phi(\omega(s))+\left[\frac{s^{6}}{2 f(s)^{4}}\left(\frac{f(s)^{3}}{s^{3}}-\omega(s)\right)+\varphi(\omega(s))\right]\left(\frac{f(s)^{3}}{s^{3}}-\omega(s)\right) .
$$

On the other hand, from relation (iv) of Theorem 3.3 one can verify that for every $i=1,2, \ldots, L$ it follows that

$$
\llbracket K \rrbracket\left(s_{i}\right)=\llbracket \Phi \circ \omega \rrbracket\left(s_{i}\right)-\frac{\varphi\left(\omega\left(s_{i}^{+}\right)\right)+\varphi\left(\omega\left(s_{i}^{-}\right)\right)}{2} \llbracket \omega \rrbracket\left(s_{i}\right),
$$

where for any function $G: \mathbf{R} \rightarrow \mathbf{R}^{m}$ having only discontinuities of the second type,

$$
\llbracket G \rrbracket(s)=G\left(s^{+}\right)-G\left(s^{-}\right) \text {. }
$$

Therefore by (5.2) and (5.3) we have proved the following result.

THEOREM 5.2. Let $f(s)$ satisfy all the conditions of Theorem 3.3 and let $\mathbf{u}$ be defined by (3.3). Then it follows that

$$
E\left(t, \mathbf{u} ; B_{\rho}\right)-E\left(0, \mathbf{u} ; B_{\rho}\right)=-\frac{4 \pi t^{3}}{3} \sum_{i=1}^{L} s_{i}^{3} \llbracket K \rrbracket\left(s_{i}\right),
$$

where $\llbracket K \rrbracket\left(s_{i}\right)$ is given by formula $(5.3)$.

Formulas similar to (5.4) have been well known in the literature on the systems of conservation laws; we only refer to [2], [3], [7], and [11]. Unlike the case considered in [9], solutions constructed here do not generally satisfy the following energy reduction criterion:

$$
E\left(t, \mathbf{u} ; B_{\rho}\right)<E\left(0, \mathbf{u} ; B_{\rho}\right) .
$$

From (5.4) it is quite clear that the possible failure of (5.5) is caused by phase transitions in the solutions, a case that is much different from that discussed in [9]. Nevertheless, in some circumstances, we can still use formula (5.4) to get some information about the energy changes of the cavitation solutions we constructed in $\S 4$.

In what follows, we use the same notations as in $\S 4$. We first prove the following result, which corresponds to Case I in $\S 4$.

Proposition 5.3. Let $\mathbf{u}$ be a cavitation solution to $(1.1)-(1.3)$ as defined in Case I of $\S 4$.

(i) If $m_{1}(\sigma)=(f(\sigma) / \sigma)^{3}$, then $E\left(t, \mathbf{u} ; B_{\rho}\right)<E\left(0, \mathbf{u} ; B_{\rho}\right)$.

(ii) Suppose $m_{2}(\sigma)=(f(\sigma) / \sigma)^{3}$. Then, if $\Phi(C)<\Phi(A)$, it follows that

$$
E\left(t, \mathbf{u} ; B_{\rho}\right)>E\left(0, \mathbf{u} ; B_{\rho}\right) \text {; }
$$

and if $\Phi(A) \leq \Phi(C)$ and $s_{1}>0$ (cf. (4.4)) is sufficiently small, the following energy reduction criterion holds:

$$
E\left(t, \mathbf{u} ; B_{\rho}\right)<E\left(0, \mathbf{u} ; B_{\rho}\right) .
$$


Proof. The conclusions follow from the basic assumption (C), (5.3), and (5.4). We omit the details but only remark that in part (ii) it suffices to choose $s_{1}>0$ so small that

$$
\llbracket K \rrbracket(s)>0, \quad 0<s \leq s_{1} .
$$

This condition is determined by the solution of problem (4.1) and thus only depends on $\mu>0$.

Case II considered in $\S 4$ renders more complicated situations about the changes of total energy for the cavitation solutions defined by (4.5) and (4.12). As indicated in the proof of the previous proposition, the general consideration of the energy changes in this case relies on the more delicate analyses on the global geometric shape of the graph of $\varphi$ and the solutions of the O.D.E.s involved. We do not intend to give a complete discussion on this issue; instead we shall present the following result, which states that under some condition solutions defined by (4.5) and (4.12) do reduce the total energy as time evolves.

Proposition 5.4. Let $\mathbf{u}$ be a cavitation solution to (1.1) defined by (4.5) or (4.12) in Case II of $\S 4$. Suppose $\Phi(A) \leq \Phi(C)$ and $s_{1}>0$ satisfies (5.6). If $s_{2}-s_{1}>0$ is sufficiently small, then

$$
E\left(t, \mathbf{u} ; B_{\rho}\right)<E\left(0, \mathbf{u} ; B_{\rho}\right) .
$$

Proof. The proof is similar to that of Proposition 5.3 and thus is omitted.

Finally, we close this paper by pointing out that it follows from Propositions 5.3 and 5.4 that

THEOREM 5.5. If $\Phi(A) \leq \Phi(C)$, then there always exist cavitation solutions of (1.1)(1.3) that reduce the total energy.

\section{REFERENCES}

[1] J. M. Ball, Discontinuous equilibrium solutions and cavitation in nonlinear elasticity, Phil. Trans. Roy. Soc. London A 306, 557-611 (1982)

[2] C. M. Dafermos, Hyperbolic systems of conservation laws, in Systems of Nonlinear Partial Differential Equations (J. M. Ball, ed.), D. Reidel, Dordrecht, 1983, pp. 25-70

[3] R. J. DiPerna, Convergence of approximate solutions to conservation laws, Arch. Rational Mech. Anal. 82, 27-70 (1983)

[4] J. L. Ericksen, Equilibrium of bars, Journal of Elasticity 5, 191-201 (1975)

[5] J. K. Hale, Ordinary differential equations, 2nd ed., R. E. Krieger Pub. Co., 1980

[6] R. Hardt, D. Kinderlehrer, and F. Lin, Existence and partial regularities of static liquid crystal configurations, Comm. Math. Phys. 105, 547-570 (1986)

[7] R. D. James, The propagation of phase boundaries in elastic bars, Arch. Rational Mech. Anal. 73, 125-158 (1980)

[8] P. D. Lax, Shock waves and entropy, in Contributions to Functional Analysis (E. A. Zarantonelo, ed.), Academic Press, New York, 1976, pp. 603-634

[9] K. A. Pericak-Spector and S. J. Spector, Nonuniqueness for a hyperbolic system: Cavitation in nonlinear elastodynamics, Arch. Rational Mech. Anal. 101, 293-317 (1988)

[10] M. Slemrod, Dynamics of first order phase transitions, in Phase Transformations and Material Instabilities in Solids (M. E. Gurtin, ed.), Academic Press, New York, 1984

[11] J. Smoller, Shock waves and reaction-diffusion equations, Springer-Verlag, New York, Berlin, Heidelberg, 1983

[12] L. Wheeler, $A$ uniqueness theorem for the displacement problem in finite elastodynamics, Arch. Rational Mech. Anal. 63, 183-189 (1976) 